thickness and collagen accumulation were determined by histological analyses. The expression of several inflammatory and pro-fibrotic mediators were measured by quantitative RT-PCR.

Results: We found that bleomycin-induced dermal thickness and lipodystrophy were increased in MKP-1 deficient mice. Collagen accumulation in the dermis and $\mathrm{mRNA}$ expression of collagens $1 \mathrm{~A} 1$ and $3 \mathrm{~A} 1$ were enhanced in the skin from MKP-1 deficient mice as compared to the skin from WT animals. Affected skin from MKP-1 deficient mice presented increased expression of factors related to inflammation and fibrosis, namely IL-6, TGF- $\beta 1$, fibronectin- 1 and YKL-40 as well as chemokines MCP-1, MIP-1 $\alpha$ and MIP-2.

Conclusions: This study demonstrates, for the first time, that MKP-1 deficient mice develop more severe bleomycin-induced dermal fibrosis than their WT counterparts, indicating that MKP-1 regulates the inflammatory and fibrotic processes typical for experimentally-induced scleroderma. These findings suggest that compounds which enhance expression/activity of MKP-1 have potential as novel drugs for the stage-specific modulation of the pathogenesis of scleroderma. References:

[1] Korhonen R, Moilanen E. Mitogen-activated protein kinase phosphatase 1 as an inflammatory factor and drug target. Basic Clin Pharmacol Toxicol. 2014; 114:24-36.

[2] Yamamoto $\mathrm{T}$ et al. Animal model of sclerotic skin. I: Local injections of bleomycin induce sclerotic skin mimicking scleroderma. J Invest Dermatol. 1999; 112:456-62.

Disclosure of Interest: None declared

DOI: 10.1136/annrheumdis-2017-eular.4816

\section{SAT0322 ADAM-17 IS EXPRESSED IN THE INFLAMMATORY MYOPATHY, AND IS INVOLVED WITH INTERSTITIAL LUNG DISEASE (ILD)}

A. Nishimi, T. Isozaki, S. Nishimi, S. Ishii, T. Tokunaga, H. Furuya,

K. Wakabayashi, T. Kasama. Division of Rheumatology, Department of Medicine, Showa University School of Medicine, Tokyo, Japan

Background: A disintegrin and metalloprotesase (ADAM) family is protease that is thought to have an important role in tissue destruction and inflammatory reaction. ADAMs are also involved in the amputation from the cell surface of inflammatory cytokines. ADAM-17 is one of the ADAM family, and is first described as the protease responsible for tumor necrosis factor (TNF)- $\alpha$ shedding. The implication of ADAM-17 substrates in immunoregulation has made this enzyme an efficient therapeutic target in the treatment of a number of pathological conditions including airway inflammation and arthritis.

Objectives: The function of ADAM-17 in myositis is unclear. Therefore, we clarify the expression of ADAM-17 in inflammatory myopathy and the role of inflammation in interstitial lung diseases (ILD).

Methods: The serum were collected from the patients who were diagnosed with inflammatory myopathy in Showa University Hospital from 2003 to 2015. Twenty-six patients were diagnosed with polymyositis (PM), 34 patients were diagnosed with dermatomyositis (DM), and 10 patients were diagnosed clinically amyopathic dermatomyositis (CADM). Clinical manifestations and clinical data were also collected. The levels of ADAM-17 in the serum samples were measured using enzyme-linked immunosorbent assay (ELISA). ADAM-17 expression was determined in muscle tissues from DM using immunohistological staining. To determine that the role of lung fibrosis in inflammatory myopathy with ILD, we used human lung fibroblasts (HLF). ADAM-17 expression on HLF was also demonstrated by immunohistrogical staining. ADAM-17 expression in interleukine (IL)-6 and IL-6 receptor (IL-6R) stimulated HLF was performed by ELISA.

Results: ADAM-17 in inflammatory myopathy was significantly higher than in healthy control $(n=19)$ (mean \pm SEM; $1048 \pm 312 \mathrm{pg} / \mathrm{ml}$ and $36 \pm 18 \mathrm{pg} / \mathrm{ml}$, respectively, $\mathrm{p}<0.05)$. ADAM-17 in corticosteroid and/or immunosuppressant treatment patient serum was also significantly decreased compared with in pre treatment patient serum $(1465 \pm 562 \mathrm{pg} / \mathrm{ml}$ and $1059 \pm 503 \mathrm{pg} / \mathrm{ml}$, respectively, $\mathrm{p}<0.01)$. In addition, ADAM-17 in inflammatory myopathy with ILD patients $(n=46)$ was significantly higher than in non-ILD patients $(n=24)(1379 \pm 454 \mathrm{pg} / \mathrm{ml}$ and $413 \pm 226 \mathrm{pg} / \mathrm{ml}$, respectively, $\mathrm{p}<0.05$ ), while ADAM-15 did not have the differences between ILD and non-ILD group. Finally, we found the expression of ADAM-17 in muscle biopsy tissue. Hence, ADAM-17 on HLF was expressed by immunohistochemistry. ADAM-17 in IL-6 and IL-6R stimulated HLF was significantly higher compared with non-stimulated $\mathrm{HLF}(48 \pm 6 \mathrm{pg} / \mathrm{ml}$ and $0 \pm 0$ $\mathrm{pg} / \mathrm{ml}$, respectively, $\mathrm{p}<0.05)$.

Conclusions: ADAM-17 is expressed in inflammatory myopathies especially with ILD and expressed on HLF, suggesting that ADAM-17 may play the role in lung fibrosis. ADAM-17 may be a potential target in inflammatory myopathies with ILD.

Disclosure of Interest: None declared

DOI: 10.1136/annrheumdis-2017-eular.3402

\section{SAT0323 THE ENDOTHELIAL-TO-MESENCHYMAL TRANSITION (ENDOMT) IN SCLERODERMA CAN BE PREVENTED BY THE USE OF DUAL ENDOTHELIN RECEPTOR ANTAGONISTS BOSENTAN AND MACITENTAN}

C. Corallo $^{1}$, M. Cutolo $^{2}$, S. Soldano ${ }^{2}$, A. Montella ${ }^{1}$, C. Chirico ${ }^{1}$, R. Nuti ${ }^{1}$,

N. Giordano ${ }^{1 .}{ }^{1}$ Medicine, Surgery and Neurosciences, University of Siena,
Siena; ${ }^{2}$ Research Laboratory and Academic Division of Clinical Rheumatology, Department of Internal Medicine, Institute for Research and Health Care (IRCCS), University of Genoa, Genoa, Italy

Background: Systemic sclerosis (SSc) is characterized by early vascular abnormalities and subsequent fibroblast activation and differentiation into myofibroblasts, leading to fibrosis. Recently, endothelial-to-mesenchymal transition (EndoMT), a complex biological process in which endothelial cells lose their specific markers and acquire a mesenchymal or myofibroblastic phenotype, has been reported in SSc.

Objectives: The goal of the study was to evaluate the potential of endothelin-1 (ET-1) dual receptor antagonists bosentan (BOS) and macitentan (MAC) to antagonize EndoMT in vitro.

Methods: 20 patients with limited SSc were enrolled and underwent double skin biopsy (affected and nonaffected skin). Fibroblasts and microvascular endothelial cells (MVECs) were isolated from biopsies. Mono- or coculture of MVECs (isolated from nonaffected skin) with fibroblasts (isolated from affected skin and stimulated with ET-1 and transforming growth factor beta [TGF- $\beta$ ]) were performed. In cocultures, the MVEC layer was left undisturbed or was preincubated with either BOS or MAC. After $48 \mathrm{~h}$ of coculture, MVECs were analyzed for their capillary formation ability and for messenger RNA and protein expression of different vascular (CD31, vascular endothelial growth factor-A [VEGF-A], VEGF-A165b) and profibrotic (alpha-smooth muscle actin [ $\alpha-S M A]$, collagen type I [Col I], TGF- $\beta$ ) molecules.

Results: MVECs showed a reduced capillary formation ability when cocultured with SSc fibroblasts with respect to mono cultures. CD31 and VEGF-A resulted in downregulation, while VEGF-A165b, the antiangiogenic isoform, resulted in upregulation. At the same time, mesenchymal markers $\alpha$-SMA, Col I, and TGF- $\beta$ resulted in overexpression in MVECs. Capillary formation ability was restored when MVECs were preincubated with BOS or MAC, also reducing the expression of mesenchymal markers and restoring CD31 expression as well as the imbalance between VEGF-A and VEGF-A165b.

Conclusions: BOS and MAC seem able to antagonize EndoMT phenomenon in MVECs in vitro. Blocking EndoMT is important for two reasons: first, because capillary formation ability in MVECs can be restored; second, because the endothelium-derived fibrotic development in SSc can be counteracted.

Acknowledgements: Thanks to Prof. Bashar Kahaleh and to Dr. Yongqing Wang from University of Toledo Medical Center, Division of Rheumatology and Immunology, OH, USA, for providing SSc microvascular endothelial cells.

Disclosure of Interest: None declared

DOI: 10.1136/annrheumdis-2017-eular.5173

\section{SAT0324 INCREASED FREQUENCIES OF CIRCULATING CXCL10-, CXCL8- AND CCL4-PRODUCING MONOCYTES AND SIGLEC-3-EXPRESSING MYELOID DENDRITIC CELLS IN SYSTEMIC SCLEROSIS PATIENTS}

T. Carvalheiro $^{1,2,3}$, S. Horta ${ }^{1,4}$, J.A. van Roon ${ }^{2,3}$, M. Santiago ${ }^{5}$, M.J. Salvador ${ }^{5}$, T.R. Radstake 2,3, H. Trindade ${ }^{1}$, J.A. da Silva ${ }^{5,6}$, A. Paiva ${ }^{1,7} .{ }^{1}$ Blood and Transplantation Center of Coimbra, Portuguese Institute of Blood and Transplantation, Coimbra, Portugal; ${ }^{2}$ Department of Rheumatology \& Clinical Immunology; ${ }^{3}$ Laboratory of Translational Immunology, University Medical Center Utrecht, Utrecht, Netherlands; ${ }^{4}$ Department of Chemistry, University of Aveiro, Aveiro; ${ }^{5}$ Department of Rheumatology, Coimbra University Hospital Center; ${ }^{6}$ Faculty of Medicine, University of Coimbra; ${ }^{7}$ Flow Cytometry Unit, Clinical Pathology Service, Coimbra University Hospital Center, Coimbra, Portugal

Background: Systemic sclerosis (SSc) is an inflammatory and fibrotic disease characterized by vascular dysfunction, excessive extracellular matrix deposition and immune dysregulation. Recent observations suggest that monocytes and dendritic cells (DCs) might be involved in SSc; including cell recruitment, trafficking, activation and an enhanced pro-fibrotic phenotype. Hence these cells might be important contributors to the disease pathogenesis. However, detailed analysis of circulating monocytes and DCs in SSc in relationship to disease activity has not been performed so far.

Objectives: To investigate the ex vivo pro-inflammatory properties of classical and non-classical monocytes as well as myeloid dendritic cells (mDCs) in SSc patients in relationship to disease activity.

Methods: This study enrolled 43 SSc patients, 30 classified as limited cutaneous $\mathrm{SSc}$ (IcSSc) and 13 as diffuse cutaneous (dcSSc). The healthy control group (HC) included 20 age- and gender- matched individuals. The Spontaneous production of CXCL10, CCL4, CXCL4 and IL-6 was intracellularly evaluated in classical and non-classical monocytes and Siglec-3-expressing mDCs from peripheral blood using flow cytometry. In addition, the production of these cytokines was determined upon toll like receptor 4 (TLR4) plus Interferon- $\gamma($ IFN- $\gamma)$ in vitro stimulation.

Results: The frequency of non-classical monocytes spontaneously producing CXCL10 was increased in both IcSSc and dcSSC subsets of SSc patients $(p<0.05)$ and CCL4 was augmented in the dcSSc patient subset $(p<0.05)$. The proportion of CCL4 producing- mDCs were also elevated in dcSSc patients $(p<0.01)$ and the percentage of mDCS producing CXCL10 only in IcSSc patients $(\mathrm{p}<0.05$ compared to $\mathrm{HC}$, but $\mathrm{p}<0.01$ comparing to dcSSc). Upon in vitro stimulation the frequency of non-classical monocytes expressing CXCL8 was 
increased in both patient groups $(p<0.01)$ and mDCs expressing CXCL8 only in IcSSc $(p<0.01)$. SSc patients characterized by the presence or history of lung fibrosis, displayed a higher frequency of non-classical monocytes expressing CCL4 and CXCL10 in dcSSc patients as compared to those without this clinical manifestation $(p<0.01$ and $p<0.05$ respectively). Strikingly, the percentage of classical monocytes producing CXCL8 was augmented upon in vitro stimulation in IcSSc patients with lung fibrosis as compared to those without $(p<0.01)$. No differences were found in the percentage of IL-6 producing cells.

Conclusions: These data point towards a role of activated non-classical monocytes and $\mathrm{mDCs}$ producing enhanced levels of proinflammatory cytokines in SSc, potentially contributing to lung fibrosis.

Acknowledgements: TC is supported by a grant from the Portuguese national funding agency for science, research and technology: Fundação para a Ciência e a Tecnologia [SFRH/BD/93526/2013]

Disclosure of Interest: None declared

DOI: 10.1136/annrheumdis-2017-eular.5050

\section{SATURDAY, 17 JUNE 2017 Scleroderma, myositis and related syndromes}

\section{SAT0325 NAILFOLD CAPILLAROSCOPIC CHANGES IN PATIENTS WITH IDIOPATHIC AND SYSTEMIC SCLEROSIS-RELATED PULMONARY ARTERIAL IPERTENSION}

A. Corrado $^{1}$, M. Correale ${ }^{2}$, N. Mansueto ${ }^{1}$, A. Mele ${ }^{1}$, A. Carriero ${ }^{1}$, R. Colia ${ }^{1}$, M. Di Biase ${ }^{3}$, F.P. Cantatore ${ }^{1} .{ }^{1}$ Department of Medical and Surgical Sciences Rheumatology Clinic; ${ }^{2}$ Department of Medical and Surgical Sciences Cardiology Clinic; ${ }^{3}$ Department of Medical and Surgical Sciences - Cardiology Clinic, University of Foggia, Foggia, Italy

Background: Pulmonary arterial hypertension (PAH) represents one of the main clinical expression of the vascular changes in Systemic Sclerosis (SSc). Many clinical and experimental evidences suggest that lung microvascular changes play a role in the pathogenesis of idiopathic PAH (IPAH) also.

Objectives: The aim of this study is to investigate the presence of capillaroscopic abnormalities in patients with idiopathic PAH and to evaluate the differences in capillary naifold changes between patients with IPAH and SSc patients with and without PAH.

Methods: 37 subjects with SSc (of whom 17 with PAH), 21 subjects with IPAH and 20 healthy subjects were recruited. PAH was diagnosed by right heart chateterization. Periungual capillaroscopy was performed in all recruited subjects, considering the following parameters: loops length and width, capillary density, microhemorrhages, avascular areas, neoangiogenesis. To define the pattern of capillary changes in IPAH and healthy subjects a semiquantitative scoring (normal, minor abnormalities, major abnormalities) was used, whereas in SSc subjects the capillary changes were defined as early, active and late pattern.

Results: In all SSc subjects a capillaroscopic scleroderma pattern was found. Particularly, comparing SSc-PAH vs SSc-nonPAH we found an early pattern in $26,7 \%$ vs $50 \%$, an active pattern in $66,6 \%$ vs $33,3 \%$ and a late pattern in a 6,6 vs $16,7 \%$ of subjects. None of IPAH subject presented a capillaroscopic scleroderma pattern, but interestingly in $36,4 \%$ of minor or major capillaroscopic changes were found. Analysing the single capillarocopic parameters, capillary density was lower in SSc subjects compared to the other groups; in SSc-PAH was lower than in SSc-nonPAH; in IPAH capillary density was lower compared to healthy control. Capillary width was higher in SSc patients compared to healthy and IPAH subjects, being higher in SSc-PAH compared to SSc-nonPAH. The number of megacapillaries, bushy capillaries and microhemorrages was significantly higher in patients with SSc-PAH compared to SSc-nonPAH patients. Interestingly, compared to healthy controls, the IPAH subjects presented a significantly lower capillary density and a significantly higher mean capillary width. Further, IPAH subjects presented a significant increase of number of microhemorrages and ectasic capillaries compared to healthy controls.

Conclusions: Microcirculation alterations, and particularly the reduction of capillary density and the increase od capillary width appears to be more severe in SSc subjects with PAH compared to SSc subjects without PAH. Capillaroscopic changes can be present in IPAH subjects also. These data support the hypothesis that in SSc peripheral microcirculation changes can be related to the entity of pulmonary microcirculation changes and that an altered vascular lung remodelling could play a role in IPAH also.

\section{References:}

[1] Cutolo $\mathrm{M}$ et al. The contribution ofcapillaroscopy to the differential diagnosis of connective autoimmune diseases. Best Pract Res Clin Rheumatol. 2007 Dec;21(6):1093-108.

[2] Riccieri V. Systemic sclerosis patients with and without pulmonary arterial hypertension: a nailfoldcapillaroscopy study. Rheumatology (Oxford). 2013 Aug;52(8):1525-8.

Disclosure of Interest: None declared

DOI: 10.1136/annrheumdis-2017-eular.5091

\section{SAT0326 THE ASSCOCIATION OF SERUM TYPE 1 INTERFERON ACTIVITY AND AUTOANTIBODIES IN INFLAMMATORY MYOSITIS}

A. Maruyama, N. Ono, M. Sakai, Y. Sadanaga, S. Koarada, Y. Tada. Rheumatology, Saga universitiy, Saga, Japan

Background: Recent reports had shown that most of clinically amyopathic dermatomyositis (CADM), which showed poor prognosis, was positive for antiMelanoma differentiation-associated gene 5 (MDA5) antibody (Ab). It had been shown that patients not only with lupus but with dermatomyositis (DM) also showed increased type 1 interferon (IFN) signature. MDA5 acts as a cytosolic RNA sensor, which drives type 1 IFN production. These facts suggested that type 1 IFN might have some roles in anti-MDA5 Ab positive patients.

Objectives: We evaluated the association of serum type 1 IFN signature and autoantibodies in patients with inflammatory myositis, in particular anti-MDA5 $A b$ positive patients and anti-aminoacyl-tRNA synthetases (ARS) Ab positive patients.

Methods: Sera from 33 inflammatory myositis patients (13 DM, 10 PM and 10 CADM) were studied for type 1 IFN activity, using a functional reporter cell assay. Briefly WISH cells were incubated with serum containing media for 6 hours. Serum IFN signature scores of the incubated cells were evaluated by the sums of gene expressions of Mx1, IFIT3, IFI44L and IFI44 by real time PCR (Reference). Anti-MDA5 $A b$ and Anti-ARS Ab were measured by ELISA. We divided these patients into three groups, anti-MDA5 Ab positive group (MDA5 group), anti-ARS $A b$ positive group (ARS group) and double negative group (DN group). We included double positive patients into MDA5 group. The presence of interstitial lung disease (ILD) and the prognosis were also investigated.

Results: MDA5 group had 12 patients (8 CADM and 4 DM), ARS group had 8 patients (4 DM, 1 CADM and 3 PM), and DN group had 13 patients (5 DM, 1 CADM and 7 PM). 9 of MDA5 group, 5 of ARS group, and 1 of DN group were complicated with ILD. Serum IFN signature scores of MDA5 group were significant higher than those of ARS group and DN group (12.43 1.406, 2.407, $\mathrm{p}=0.0005$ ). The most of ARS group showed low serum IFN signature activities. The overall survivals of ARS group were fairer than MDA5 group, but not significantly. The deceased cases of MDA5 group showed especially high serum IFN signature activities.

Conclusions: We characterized two major groups in inflammatory myositis patients. ARS group was characterized by low IFN signature with the susceptibility to DM and ILD. MDA5 group was characterized by high serum IFN signature with the high susceptibility to CADM. Our results suggest that these two entities may have different onset mechanisms, leading to different outcomes.

References:

[1] Niewold TB, Rivera TL, Buyon JP, Crow MK. Serum type I interferon activity is dependent on maternal diagnosis in anti-SSA/Ro-positive mothers of children with neonatal lupus. Arthritis Rheum. 2008 Feb;58(2):541-6.

Disclosure of Interest: None declared

DOI: 10.1136/annrheumdis-2017-eular.4900

\section{SAT0327 NUTRITIONAL STATUS IN PATIENTS WITH SYSTEMIC SCLEROSIS}

A. Wojteczek ${ }^{1}$, M. Ziętkiewicz ${ }^{1}$, S. Małgorzewicz ${ }^{2}$, Z. Zdrojewski ${ }^{1}{ }^{1}{ }^{1}$ Department of Internal Medicine, Connective Tissue Diseases and Geriatrics; ${ }^{2}$ Department of Clinical Nutrition, Medical University of Gdańsk, Gdańsk, Poland

Background: Systemic sclerosis (SSc) is a chronic connective tissue disease characterized by involvement of multiple organs. Many clinical aspects, such as gastrointestinal involvement, mood disturbances, functional status, and inflammation, may lead to disease-related malnutrition [1]. The connection between inadequate nutritional status and systemic sclerosis is still not well established. It is important to identify the symptoms of malnutrition, because it is known as a predictor of poor clinical outcome [2].

Objectives: To assess nutritional status in patients with systemic sclerosis.

Methods: The study involved fifty-two patients with SSc (44 women and 8 men, mean age $54,3 \pm 11,7$ year) who were diagnosed according to ACR/EULAR criteria. The assessment of nutritional status was determined by subjective global assessment (SGA), body mass index (BMI) and level of serum albumin. Appetite was assessed by simplified nutritional appetite questionnaire (SNAQ). In all patients hand grip strength and triceps skinfold were established. The C-reactive protein (CRP), lipid profile, and level of haemoglobin/lymphocytes were measured in serum.

Results: Inadequate nutritional status was diagnosed in 14 patients $(26,9 \%)$ with SSc. According to SGA $11(21,15 \%)$ patients had signs of mild malnutrition, while $41(78,85 \%)$ were well-nourished. Considering BMI, 1 patient $(1,92 \%)$ was underweight, $24(46,15 \%)$ were eutrophic, $21(40,38 \%)$ overweight and 6 $(11,54 \%)$ obese. Significantly lower BMI had patients with inadequate nutritional status $(23,17 \pm 4,47$ vs. $25,98 \pm 3,34 ; p=0,009)$. Low level of serum albumin was detected in 5 patients $(9,6 \%)$ with SSc. Level of serum albumin and appetite were significantly decreased in patients with inadequate nutritional status $(p=0,009$; $p=0,003$ ). No statistical differences were noticed in hand grip strength, triceps skinfold, lipid profile, levels of CRP and haemoglobin/lymphocytes.

Conclusions: Malnutrition in systemic sclerosis is still underestimated clinical issue. This study provides useful data about nutritional status of patients with 